\title{
Stephan Gerhard Huber
}

Institut für Bildungsmanagement und Bildungsökonomie (IBB) der Pädagogischen Hochschule Zug

\section{Paula Mork}

Institut für Bildungsmanagement und Bildungsökonomie (IBB) der Pädagogischen Hochschule Zug

\section{Wertschätzung zeigen und erfahren}

\section{Ausgewählte Aspekte aus wissenschaftlicher und schulpraktischer Sicht}

DOI: https://doi.org/10.53349/10.53349/sv.2021.i2.a106

\begin{abstract}
Wertschätzung ist eine grundlegende berufsethische Prämisse im schulischen Bildungskontext und integraler Bestandteil von pädagogischem Handeln schlechthin. Vor diesem Hintergrund kommt Mitarbeitenden an Schulen und im Schul- und Bildungssystem in ihrer (gesellschaftlichen) Aufgabe der Sicherung und Weiterentwicklung der Qualität von Bildung mit dem Ziel, Kinder und Jugendliche bestmöglich in ihrer Persönlichkeitsentwicklung in einem sozialen Zusammenhang zu fördern, eine bedeutende Rolle zu. Im Rahmen ihrer Funktion, Rolle und Aufgaben und vor dem Hintergrund ihrer Qualifikationen, Motivationen, Kompetenzen, der Rahmenbedingungen und der schulspezifischen Situation leisten sie einen wichtigen Beitrag in diesem Bildungsprozess. ${ }^{1}$
\end{abstract}

Wertschätzung, Vertrauen, Fürsorge, Berufsethos, affektives Commitment, Responsible Leadership

Wertschätzung hat verschiedene Facetten und kann im professionellen Geschehen wissenschaftlich unterschiedlich ge- und erfasst werden. Es geht nicht nur um das Loben und Anerkennen von Leistung(en). Wertschätzung spiegelt sich auch im Erleben von Aufmerksamkeit, im Erhalten von konstruktivem Feedback, im Erleben von sozialer Unterstützung, im Ausüben sinnvoller Tätigkeiten, dem passgenauen Einsatz im Hinblick auf Aufgaben und Kompetenzen. Sie zeigt sich auch in der Persönlichkeit und Organisationskultur, im Erleben von positiven Sozialdynamiken.

In einem professionellen Kontext gehört Wertschätzung zu den Personalmanagementaufgaben in doppelter Perspektive: Es geht zum einen um Führungsverhalten von der Schulaufsicht den Schulleitungen gegenüber und von der Schulleitung den schulischen Mitarbeitenden ge- 
genüber. Es geht aber auch um die Wertschätzung den Führungskräften gegenüber, jeweils von der übergeordneten Ebene sowie den jeweiligen Mitarbeitenden.

Bei der Wertschätzung kommen Aspekte wie der Personaleinsatz, die Personalentwicklung, Personalbeurteilung, Personalführung, Personalunterstützung zum Tragen, es geht aber auch um einen professionellen Umgangston und eine respektvolle Haltung der Arbeit und allen Akteuren gegenüber. Wertschätzung ist unter anderem assoziiert mit Anerkennung, Fordern und Fördern, Leistung, Zutrauen, Vertrauen, affektivem Commitment, Fürsorge und Respekt.

Gerade im pädagogischen Bereich ist Wertschätzung auch verbunden mit berufsethischen Aspekten (Ruep \& Schratz 2012, S. 14). Dieses professionelle Ethos, auf das auch Schärer und Zutavern (2018) rekurrieren, soll dazu führen, dass fair und fürsorglich gehandelt wird.

Im Folgenden wird eine Annäherung an den Begriff Wertschätzung im Bildungskontext versucht, indem exemplarische Überlegungen aus der Organisationspädagogik, Konzeptionen und berufsethische Standards aus der Bildungspraxis und -forschung sowie Strategien einer Responsible Leadership präsentiert werden, um abschließend daraus Strategien einer kooperativen Führung abzuleiten.

\section{Überlegungen aus der Organisationspädagogik}

Wenn Schule als Institution erzieht, muss sie „ein Modell dafür sein, wozu sie erzieht“ (Rosenbusch 2005, S. 11). Die Ziele einer Schule, so zum Beispiel ein wertschätzender Umgang miteinander, müssen in ihrer alltäglichen Praxis erfahrbar sein, da praktische Erfahrung lernwirksamer ist als kognitive Wissensvermittlung zum selben Sachverhalt. Für die Schule als Organisation bedeutet dies, dass Mündigkeit, Anerkennung, Kooperation und Selbsttätigkeit in ihr erlebbar, ihre Struktur und Kultur also auf diese Ziele abgestimmt sein müssen. Indem die Menschen in der Schule als mündig angesehen und angesprochen werden, in ihren Stärken wahrgenommen und ihnen auf Augenhöhe begegnet wird, wird Wertschätzung deutlich.

Pädagogische Führung spielt bei der Realisierung der Ziele einer wertschätzenden Schulkultur eine entscheidende Rolle. Schulleitungshandeln als pädagogisches Organisationshandeln ist mit einem normativen Anspruch verbunden. Hier liegen unter anderem folgende Handlungsmaxime nahe (Rosenbusch 1997, 2013):

- Pädagogische Zielvorstellungen haben Vorrang vor Verwaltungsaspekten; Verwaltung hat eine dienende Funktion.

- In der pädagogischen Arbeit wird eine (erwachsenen-)pädagogische Praxis sichtbar, die zugleich als Vorbild für die (kind-)pädagogische Praxis und das Lernen der Schüler*innen dient.

- Es gilt das Prinzip der Schatzsuche statt eines Fahndens nach Defiziten. 


\section{Konzeptionen und berufsethische Standards aus der Bildungspraxis und -forschung}

In der allgemeinen Führungsforschung wird unter dem Stichwort des 'Moral Leadership' (Sergiovanni 2005; Fullan 2010) insbesondere dem Aspekte moralischen Führungshandelns Bedeutung zugesprochen. So argumentieren z.B. Hannah, Lester und Vogelsang "that the developmental experiences and processes that faciliate authenticity, such as heightended cognitive complexity and self-wawareness in the leader, foster higher levesl of moral reasoning and reflection, which in turn positively influence the leader and ultimately the followers' moral behaviour" (Hanna, Lester \& Vogelsang 2005, S. 44). Insbesondere der mögliche moralische Einfluss auf die 'Follower' erscheint für den schulischen Kontext interessant. Doch wie kann ein solcher Effekt aus der Personenabhängigkeit einzelner Führungspersonen gelöst und z.B. schulübergreifend erzielt werden? Eine mögliche Antwort lautet: Durch das Formulieren von (berufs-)ethischen Standards:

Im Sinne einer werteorientierten, auf berufsethischen Grundlagen fußenden pädagogischen Arbeit haben einige Länder ethische Standards als Teil ihres Professionsverständnisses entwickelt. So richten beispielsweise die Mitglieder des Ontario College of Teachers (2021) ihr berufliches Handeln und Verhalten an folgenden professionellen und ethischen Standards aus: Vertrauen, Fürsorge, Respekt und Integrität. Sie zeigen damit Verantwortung in ihren Beziehungen zu Schüler*innen, Eltern, Kolleginnen und Kollegen, Bildungspartnern, der Umwelt und der Öffentlichkeit. Die Standards, auf die auch im Aufsatz von Ruep und Schratz (2012) verwiesen wird, verfolgen das Ziel, den Wert und die Würde des Lehrerberufs zu reflektieren und aufrechtzuerhalten, die berufsethische Verantwortlichkeit und Verpflichtung aufzuzeigen, einen Orientierungsrahmen für ethische Entscheidungen und ethisches Handeln im Lehrerberuf zu geben und schließlich das Vertrauen in den Lehrerberuf zu fördern.

\section{Vertrauen}

Wertschätzung schafft Vertrauen, und Vertrauen schafft Wertschätzung. In Vertrauensgemeinschaften werden Meinungen und Haltungen geteilt (Seligman 2012; Solzbacher 2017), was ein gemeinsames Zielverständnis begünstigen dürfte. Vertrauen gilt weiter als Schlüsselelement für eine funktionierende Kommunikation (Hoy et al. 2002). Fehlt das Vertrauen, ist es unwahrscheinlich, dass Lehrpersonen sich in der eigenen professionellen Praxis auf gemeinsame Unterrichtsplanung, kollegiale Beobachtung oder reflektierenden Dialog einlassen mögen (Tschannen-Moran 2014). Vertrauen ist eine wichtige Qualität der Interaktion. Sie wird gefördert durch Wertschätzung und sie ermöglicht facettenreiche und personen- und aufgabenbezogene Wertschätzung.

\section{Fürsorge}

In den vergangenen fünf bis zehn Jahren entstanden Konzeptionen, die fürsorgliches Handeln nicht nur im sozialen Umgang miteinander propagierten, sondern Fürsorge als Grundgedan- 
ken von Führungshandeln etablierten. Fürsorge ist in den Führungskontext, in die Strategie und das Verhalten eingebettet. Die Umsetzung fürsorglicher Führung verläuft nach Seashore Louis et al. (2016) über vier konkrete Ankerpunkte:

- Die Schulgemeinschaft wird in die Vision und Herausforderung einer fürsorglichen Schule mit eingebunden.

- Die Möglichkeiten und Hintergründe der Schule in Bezug auf Fürsorge werden eingeschätzt. Die Schulgemeinschaft wird an diesem Reflexionsprozess beteiligt.

- Die Schulkultur wird fürsorglich gestaltet, indem Unterstützungssysteme etabliert und soziale Beziehungen sowie das Normen- und Wertesystem der Schule gestärkt werden.

- Fürsorgliche Beziehungen als Schulgemeinschaft werden über die Grenzen des Schulhauses hinausgetragen und fürsorgliche Partnerschaften auch außerhalb der Schule kultiviert.

Für die Mitglieder des Ontario College of Teachers (2021) umfasst der ethische Standard der Fürsorge Mitgefühl, Akzeptanz und Interesse für die Entwicklung des Potenzials der Schüler*innen. Die Mitglieder bringen ihr Engagement für das Wohlergehen und das Lernen der Schüler*innen durch von Empathie getragene aktive positive Zugewandtheit und fundierte diagnostische Kompetenz zum Ausdruck.

Bei aller Euphorie über eine möglichst fürsorglich geführte Schulgemeinschaft gilt es jedoch zu beachten, dass fürsorgliche Leitungspersonen, ebenso wie charismatische, verklärt und glorifiziert werden können. Fürsorgliche Führung wird sich schließlich immer in einem Spannungsfeld zwischen den gerechtfertigten Bedürfnissen verschiedener Mitarbeitenden-Gruppen und dem Erreichen von mittel- und langfristigen Schulentwicklungszielen bewegen (Eisner 2021). Wertschätzung ist aus unserer Sicht ein integrativer Bestandteil von Fürsorge.

\section{Respekt}

Respekt, Rüchsichtnahme und soziale Wertschätzung oder Freundschaft sind Formen der Anerkennung, mit innen sind wichtige soziale Werte im schulischen Leben beschrieben (Felder 2021, S. 8). Der von den Mitgliedern des Ontario College of Teachers (2021) definierte ethische Standard „Respekt“ beinhaltet Vertrauen und Fairness. Die Mitglieder achten die Menschenwürde, das emotionale Wohlbefinden und den jeweiligen Entwicklungsstand der Kinder und Jugendlichen. In ihrer beruflichen Praxis leben sie Respekt gegenüber Werten, sozialer Gerechtigkeit, Freiheit, Demokratie und der Umwelt vor.

\section{Integrität}

„Integrität wird häufig als Makellosigkeit, Unbescholtenheit oder Unbestechlichkeit definiert, aber auch Anständigkeit, Zuverlässigkeit, Vertrauenswürdigkeit oder Unverletzlichkeit dienen als Charakteristika. Es stammt von dem lateinischen Wort „integer" für „untadelig, unversehrt" ab" (Keller 2019). Für die Mitglieder des Ontario College of Teachers (2021) werden Ehrlichkeit, Zuverlässigkeit und moralisches Handeln durch die ethische Norm der Integrität 
verkörpert. Kontinuierliche Reflexion hilft den Mitgliedern, ihre beruflichen Verpflichtungen und Verantwortlichkeiten integer wahrzunehmen.

\section{Strategien einer Responsible Leadership}

Verantwortung für Bildung wird aus einem ethischen Denken und Handeln heraus wahrgenommen, in dem sich ein ganzheitliches Bildungsverständnis widerspiegelt und das auf die Förderung von Bildungsbiografien durch Persönlichkeitsentwicklung zielt. Bei den professionellen Akteuren im Bildungssystem setzt dies eine hohe Professionalität, ein wertebezogenes Berufsethos und eine emotionale Bindung an die Organisation bzw. ein Zugehörigkeitsgefühl (affektives Commitment), voraus.

Führungskräften kommt hierbei eine besondere Bedeutung zu. Als transformationale Führungskräfte sorgen sie dafür, dass übergeordnete Werte und Ideale entwickelt werden, schaffen eine Vision und motivieren Mitarbeitende, indem sie mitteilen, inwieweit Ziele erreicht worden sind (Huber 2005). Sie unterstützten die persönliche Entwicklung. Transformationale Führung im schulischen Kontext fokussiert die Lehrkräfte und führt zu einer hohen Identifikation mit den Entwicklungszielen der Schule. Die Schulleitung verfügt über pädagogische Werte und Visionen, die reflektiert, im Sinne einer systemischen, kohärenten und werteorientierten Qualitätsentwicklung an Schulen mit allen Beteiligten gemeinsam abgestimmt, weiterentwickelt und stets kommuniziert werden.

Die Schulleitung agiert hierbei in einer Doppelrolle: Sie steuert einerseits die Professionalisierung des schulischen Personals, was auch die erzieherische Arbeit der Lehrer*innen einschließt. Andererseits ist sie als Person selbst gefordert, eine werteorientierte Haltung einzunehmen und sich selbst sowie die Akteure im System Schule immer wieder kritisch zu hinterfragen (Huber, Schneider, Lussi, Klein \& Hader-Popp 2019). Quick misst diesem moralischen Denken und Handeln der Führungsperson eine hohe Bedeutung zu: "Leadership in any endeavor is a moral task, but even more so for educational leaders. Educational leaders are not only responsible for the success of their particular institution, but their work can impact various other institutions now and in the future, those who are led will be the future leaders of tomorrow." (Quick 2008, S. 336)

Mit Responsible Leadership werden verschiedene Handlungsstrategien verfolgt (Huber et al. 2019, siehe Ausgabe 1 von \#schuleverantworten) - verantwortungsvoll Führende handeln professionell, analytisch, moralisch, kooperativ, transformativ und sinnstiftend/kommunizierend sowie strategisch, ihr Handeln ist auch Modell dafür, wozu die Schule erziehen will.

Damit bleiben pädagogische Führungskräfte, die Verantwortung für Bildung und Erziehung übernehmen und wertebasiertes Führen als Führungsaufgabe annehmen, pädagogischen Werten verpflichtet, die den Umgang mit den Schüler*innen ebenso bestimmen wie die Kooperation mit dem Kollegium. Das pädagogische Führungshandeln, und hier ist das Führungshandeln von Lehrerinnen und Lehrern explizit mit angesprochen, ist damit wie bereits formu- 
liert auch Modell dafür, wozu die Schule erziehen will. Für die Modellwirkung ist entscheidend, wie das Leitungshandeln von den anderen Akteuren in der Schule erlebt und gedeutet wird.

Einen Ansatz einer wertschätzenden Schulentwicklung bzw. Schulleitung entwirft Olaf-Axel Burow (2016). Er definiert drei Dimensionen einer wirksamen Schulleitung:

1. Salutogense: In Anlehnung an das Konzept des israelischen Soziologen Aron Antonovsky sollte sich Schulleitung vorrangig die Frage stellen, was Lehrkräfte gesund hält und wie sie sie beim Gesundbleiben unterstützen kann. Denkbar sind dabei Unterstützungsangebote auf drei Ebenen: informationell (z.B. Ratschläge), instrumentell (z.B. Bereitstellung von Ressourcen) und emotional (z.B. Verständnis).

2. Selbstbestimmung: Als Rahmen einer wertschätzende Kommunikation zwischen den diversen Instanzen innerhalb des Schulsystems steht die Ermöglichung von Selbstbestimmung aller Beteiligten, die insbesondere durch Vernetzung und Partizipation erreicht werden kann.

3. Wertschätzung: Wertschätzung ist die dritte Dimension einer wirksamen Schulleitung, die sich organisch aus den ersten beiden Dimensionen ergibt: „Wenn wir die Kriterien von Salutogenese und Selbstbestimmungstheorie konsequent berücksichtigen, tragen wir nicht nur zur Potenzialentfaltung aller bei und steigern die Leistungsergebnisse, sondern wir schaffen damit zugleich ein Wohlfühlklima, das die Belastungsfaktoren der traditionellen Schule für Lehrer, Schüler, aber auch Eltern reduziert" (Burow 2016, S. 76).

Die Wirksamkeit eines wertschätzenden und unterstützenden Umfeldes wird u.a. durch eine Studie des bayerischen Aktionsrats "Bildung" aus dem Jahre 2014 unterstrichen. Während die Studie einerseits feststellt, dass Lehrkräfte ihren Beruf überdurchschnittlich oft als stark belastend empfinden, zeigt sie zum anderen, dass eine unterstützende Schulleitung dieser Problematik entgegenwirken kann und sich signifikant auf das Lehrer*innenengagement auswirkt: So „ergab eine Mehrebenenanalyse der Zusammenhänge zwischen Merkmalen der Schulen und Lehrerengagement, dass - nach Kontrolle individueller Lehrermerkmale - die Unterstützung durch die Schulleitung (als einziges Schulmerkmal) prädiktiv für das mittlere Lehrerengagement war: Schulen mit unterstützenden Schulleitungen hatten engagiertere Lehrkräfte." (Blossfeld et al. 2014, S. 108)

\section{Kooperative Führung gestalten}

In der Gestaltung eines Berufsethos, einer Responsible Leadership, braucht es geeignete Strukturen und Prozesse, die systemische Stimmigkeit und Kohärenz ermöglichen. Kooperative Führung - beispielsweise in einer erweiterten Schulleitung, in einer mittleren Führung oder in einer Steuergruppe - ist in diesem Sinne die erste Wahl, wenn es darum geht, gemeinsam Schule zu gestalten (Spillane 2006; Harris \& Spillane 2008; Harris 2009; Huber 2010, 2020). 
Dem Begriff der kooperativen Führung liegt die Vorstellung zugrunde, „dass die Führungsfunktion dauerhaft im Miteinander mit den Mitarbeiterinnen ausgestaltet wird und eine hohe Intensität der Entscheidungsbeteiligung aufweist" (Kansteiner-Schänzlin 2002, S. 47). Voraussetzung ist, dass Werthaltungen und Kompetenzen von Personen (Leitungspersonal und Kollegium) einerseits und Teamstrukturen andererseits so zusammenkommen, dass die Handlungsprozesse (in der Schule: Lehr-Lern- und Erziehungsprozesse) von Kooperation bestimmt werden. Dann ist systemische Stimmigkeit erreicht, denn aufgrund der komplexen Hierarchie innerhalb der Schule ist Kooperation angemessene Handlungsrationalität.

Rosenbusch (2005) begründet dies überzeugend: „,,Kollegialität trotz Hierarchie' (Rosenbusch 1994a) bezieht sich auf Formen des Umgangs Einzelner innerhalb des Kollegiums mit Vorgesetzten. Strukturell gesehen, herrscht sowohl innerhalb als auch außerhalb der Schule (in der Schulverwaltung) eine Hierarchie mit Vorgesetzten und Untergebenen, ein Schulleiter ist Vorgesetzter einer Lehrerin und eine Dezernentin die Vorgesetzte eines Schulleiters. [...] In der speziellen komplexen Hierarchie der Schule müssen wir neu überlegen. Zwar sind die gegenseitigen Verantwortlichkeiten zu respektieren, doch sollte die kollegiale Verpflichtung auf gemeinsame Ziele einschließlich der gegenseitigen persönlichen und fachlichen Wertschätzung dominieren. [...] Kollegiale Kommunikationspraxis heißt praktisch ausgedrückt: nicht ein Vorgesetzter spricht mit einem Untergebenen, sondern ein Fachmann für Schulführung mit einem Fachmann für beispielsweise Geographieunterricht" (S. 117).

\section{Fazit}

Pädagogische Führungskräfte wirken durch das, was sie sagen, aber vor allem durch das, was sie tun. Schule wird nur dann glaubwürdig wirken, wenn das Leitungshandeln in der Schule als kohärent erfahren wird. Wenn also als passend und übereinstimmend erlebt wird, was die Leitung sagt, wie sie handelt und was sie von den Mitarbeitenden und Schüler*innen fordert. Diese Glaubwürdigkeit ist grundlegend für Bindung, Verlässlichkeit und emotionale Sicherheit als Grundlage für Offenheit, Vertrauen und Lernen. Wertebasierte Führung ist eben nicht nur als „Leitung der Organisation durch die Schulleitung“ von Bedeutung, sie bestimmt auch wesentlich die pädagogischen Interaktionen zwischen Schüler*innen und Lehrpersonen (Bartz 2007, S. 2).

Die anfangs skizzierten Aspekte von Wertschätzung werden von einem bzw. einer verantwortungsvoll Führenden beachtet. Auf Grundlage einer berufsethischen Rahmung aktiv Wertschätzung zu zeigen, setzt voraus, dass die Führungskraft vom Empfänger, der Empfängerin beziehungsweise von Empfängergruppen der Wertschätzung her denkt, nicht von sich als Sender aus. Die konkrete Realisierung kann demzufolge von Organisationskultur zu Organisationskultur und von Person zu Person unterschiedlich sein. Wertschätzung zu zeigen ist ein integraler Bestandteil von Responsible Leadership. 


\section{\# schuleverantworten}

führungskultur_innovation_autonomie

\section{Literaturverzeichnis}

Bartz, A. (2007). Schulleitungshandeln als Modell: das Führungshandeln von Schulleitung und Lehrkräften gestalten. In A. Bartz, J. Fabian, S. G. Huber, C. Kloft, H. Rosenbusch \& H. Sassenscheidt (Hrsg.), PraxisWissen SchulLeitung, Kennziffer 67.14. Köln: Wolters Kluwer.

Blossfeld, H. et al. (2014). Psychische Belastugnen und Burnout beim Bildungspersonal. Empfehlungen zur Kompetenz- und Organisationsentwicklung. Münster: Waxmann. https://www.vbw-bayern.de/Redaktion/Frei-zugaengliche-Medien/Abteilungen-GS/Bildung/2014/Downloads/ARB_Gutachten_Psychische-Belastungen-und-Burnout-beim-Bildungspersonal.pdf (letzter Zugriff 24.08.2021).

Burow, A. O. (2016). Wertschätzende Schuleitung. Der Weg zu Engagement, Wohlbefinden und Spitzenleistung. Weinheim, Basel: Beltz.

Eisner, R. (2021). Schulleitung und Schule - Interviewstudie über Werte, Bedürfnisse, Anforderungen und Ziele als relevante Merkmale für die Funktionalität von schulischer Führung. Masterarbeit im Fachbereich 12 Erziehungs- und Bildungswissenschaften an der Universität Bremen.

Felder, F. (2021). Die Systematik der Anerkennung. Anmerkungen zu einem zentralen bildungswissenschaftlichen Begriff. PraxisForschungLehrer*innenBildung, 3 (2), 7-17. https://doi.org/10.11576/pflb4192

Fullan, M. (2010). Positive Pressure. In A. Hargreaves, A. Lieberman, M. Fullan \& D. Hopkins (Hrsg.), Second International Handbook of Educational Change (S. 119-130). Dordrecht et al.: Springer.

Hannah, S. T., Lester P. B., Vogelsang G. R. (2005). Moral Ladership: Explicating the Moral Component of Authetnic Leadership. In W. L. Garnder, B. J. Avolio \& F. O. Walumbwa (Hrsg.), Authentic Leadership. Theory and Pracitc: Origins, Effects and Developments (S. 43-81). Emerald.

Harris, A. (2009). Distributed Leadership: Different Perspectives. Dordrecht: Springer.

Harris; A. \& Spillane, J. (2008). Distributed leadership through the looking glass. Management in Education, Vol 22(1): S. 31-34.

Huber, S.G. (2005). Führungskonzeptionen und Führungsmodelle im Überblick. In A. Bartz, J. Fabian, S.G. Huber, C. Kloft, H. Rosenbusch \& H. Sassenscheidt (Hrsg.), PraxisWissen SchulLeitung (10.11). München: Wolters Kluwer.

Huber, S.G. (2010). System Leadership - systemische Schulentwicklung durch Kooperation. Journal für Schulentwicklung, 2, S. 8-21.

Huber, S.G. (2020). Kooperative Führung: Führungsteams initiieren, koordinieren und steuern Schulentwicklung. In S.G. Huber (Hrsg.), Handbuch für Steuergruppen. Grundlagen für die Schulentwicklung und das Schulmanagement (S. 17-36), 4. überarb. und erw. Aufl. Köln: Carl Link.

Huber, S.G. (2021). Führungskraft als Vorbild. bayerische schule 74(3), 33-35. 


\section{\# schuleverantworten}

führungskultur_innovation_autonomie

Huber, S.G., Schneider, N., Gleibs, H.E. \& Schwander, M. (2013). Leadership in der Lehrerbildung. Entwicklung von Kompetenzen für pädagogische Führung. Stiftung der Deutschen Wirtschaft und Robert Bosch Stiftung: Berlin.

Huber, S.G., Schneider, N., Lussi, I., Klein, U. \& Hader-Popp, S. (2019). Verantwortung für Bildung und Erziehung übernehmen und wertebasiertes Führen gestalten. In J. Hugo, N. Brink, J. Seidemann \& M. Drahmann (Hrsg.), Verantwortung im Kontext von Schule. Perspektiven aus Wissenschaft und Praxis (S. 117-129). Münster, New York: Waxmann.

Keller T. (2019). Definition von Integrität im Führungskontext. In: Integrität als Führungskompetenz. essentials. Springer: Wiesbaden. https://doi.org/10.1007/978-3-658-25999-0_2

Ontario College of Teachers (2021). Ehtical Standards. https://www.oct.ca/public/professional-standards/ethical-standards (letzter Zugriff 12.08.2021).

Quick, P. M., Normore, A. H. (2008). Moral Leadership in the 21st Century: Everyone Is Watching - Especially the students. In The Educational Forum 68:4 (S. 336-347).

Sergiovanni, T. J. (2005). Strengthening the heartbeat. Leading and learning together in schools. San Francisco: Jossey-Bass.

Rosenbusch, H. (2005). Organisationspädagogik der Schule. Grundlagen pädagogischen Führungshandelns. Köln: Luchterhand.

Rosenbusch, H. (2013). Organisationspädagogische Führungsprinzipien. In S.G. Huber (Hrsg.), Handbuch Führungskräfteentwicklung. Grundlagen und Handreichungen zur Qualifizierung und Personalentwicklung im Schulsystem (S. 96-103). Köln: Carl Link.

Ruep, M. \& Schratz, M (2012). Wert und Werte von Führung in der Schule. In S.G. Huber (Hrsg.), Jahrbuch Schulleitung 2012. Befunde und Impulse zu den Handlungsfeldern des Schulmanagements (S. 1117). Köln: Wolters Kluwer.

Schärer, H.-R. \& Zutavern, M. (2018) (Hrsg.). Das professionelle Ethos von Lehrerinnen und Lehrern. Perspektiven und Anwendungen. Münster, New York: Waxmann.

Seashore Louis, K., Murphy, J., \& Smylie, M. (2016). Caring Leadership in Schools: Findings From Exploratory Analyses. Educational Administration Quarterly 52(2), 310-348. doi:10.1177/0013161X15627678

Seligman, M. (2012). Flourish - Wie Menschen aufblühen. München: Kösel [Originalausgabe 2011: Flourish. A Visionable New Understanding of Happyness and Well-Being. New York: Simon \& Schuster/Free Press].

Solzbacher, C. (2017). Die professionelle pädagogische Haltung - Mit dem inneren Kompass kohärent, nachvollziehbar und situationsübergreifend handeln. In A. Bartz, M. Dammann, S.G. Huber, T. Klieme, C. Kloft \& M. Schreiner (Hrsg.), PraxisWissen SchulLeitung (53.14). München: Wolters Kluwer. 


\section{\# schuleverantworten}

führungskultur_innovation_autonomie

Spillane, J.P. (2006). Distributed Leadership. San Francisco CA: Jossey-Bass.

Tschannen-Moran, M. (2014). The interconnectivity of trust in schools. In D. Van Maele, P.B. Forsyth, \& M. Van Houtte (eds.), Trust relationships and school life: The influence of trust on learning, teaching, leading, and bridging. Springer Publisher, 57-81. DOI 10.1007/978-94-017-8014-8_3

\section{Anmerkungen}

${ }^{1}$ Der ursprünglich zugrunde liegende Beitrag aus der "bayerischen schule 3/2021", S. 33-35 (Huber 2021), wurde umfassend ergänzt und thematisch erweitert.

\section{Autor*innen}

Stephan Gerhard Huber, Prof. Dr.

Leiter des Instituts für Bildungsmanagement und Bildungsökonomie IBB der Pädagogischen Hochschule Zug, Gastprofessor „,an der JKU Linz und Dozent an den PHn OÖ, NÖ, LU, SZ, ZH, WG sowie Adjunct Professor am GIER der Griffith University Brisbane und Senior Research Fellow an der Education University of Hong Kong. Er leitet das Schul-Barometer für Deutschland, Österreich und die Schweiz (www.Schul-Barometer.net), die World School Leadership Study, den Young Adult Survey Switzerland (www.chx.ch/YASS) und das World Education Leadership Symposium (WELS.EduLead.net).

Kontakt: stephan.huber@phzg.ch

\section{Paula Mork}

Studentische Mitarbeiterin am Institut für Bildungsmanagement und Bildungsökonomie IBB der Pädagogischen Hochschule Zug. 